\title{
Co-Cr films prepared by sputtering using electron cyclotron resonance microwave plasma
}

\author{
S. Yamamoto, K. Sato, H. Kurisu, and M. Matsuura \\ Faculty of Engineering, Yamamaguchi University, Tokiwadai, Ube 755, Japan
}

The sputtering deposition using an electron cyclotron resonance (ECR) microwave plasma was tried to use in the fabrication of the $\mathrm{Co}-\mathrm{Cr}$ perpendicular magnetic recording media. As the Ar sputtering gas pressure increased from $4 \times 10^{-2}$ to $8 \times 10^{-2} \mathrm{~Pa}$, the $\mathrm{Co}(002) \mathrm{x}$-ray diffraction peak intensity increased and the half-value width of the rocking curve $\Delta \theta_{50}$ decreased. This result implies that Co-Cr films with high perpendicular orientation and good crystallinity are achieved at high Ar gas pressure. The Co-Cr films deposited at a target to substrate distance of $230 \mathrm{~mm}$ had a good preferred crystal orientation $\left(\Delta \theta_{50}\right.$ less than $\left.4^{\circ}\right)$, high perpendicular magnetic anisotropy $\left(H_{k}\right.$ higher than $4 \mathrm{kOe}$ ), and high perpendicular coercivity over 1400 Oe even though the Co-Cr thickness is as small as about $50 \mathrm{~nm}$, and no underlayers were introduced. Thus, the ECR sputtering has high potential in the deposition of the $\mathrm{Co}-\mathrm{Cr}$ films for ultrahigh density recording media. (C) 1996 American Institute of Physics. [S0021-8979(96)14608-3]

\section{INTRODUCTION}

An extremely high density recording exceeding 500 kFRPI has already been achieved in perpendicular magnetic recording using a $\mathrm{Co}-\mathrm{Cr}$ perpendicular media. ${ }^{1-3}$ It has been proven that such a high recording resolution of $\mathrm{Co}-\mathrm{Cr}$ films is originated from the compositional separation into Co- and Cr-rich regions in the size range of a few nanometers. ${ }^{4-6}$ In order to improve the recording resolution further, it is necessary to develop the film deposition technology by which the compositional separation is controlled more precisely.

Recently, an electron cyclotron resonance (ECR) microwave plasma has been developed, and used in the thin-film deposition processing. Compared with the conventional diode sputtering, the ECR sputtering features: (1) generation of dense plasma even at $\mathrm{Ar}$ gas pressure as low as $10^{-2} \mathrm{~Pa},(2)$ generation of highly ionized plasma, and its controllable irradiation to the substrate. ${ }^{7,8}$

Up to now, there have been only a few attempts concerning the application of the ECR sputtering to the $\mathrm{Co}-\mathrm{Cr}$ film deposition. ${ }^{9}$ Hirono and co-workers ${ }^{10}$ have succeeded in reducing the grain size of $\mathrm{Co}-\mathrm{Cr}$ films with a compositionally separated microstructure, while maintaining high grade magnetic properties by using the ECR sputtering method.

In this study, we have tried the deposition of the $\mathrm{Co}-\mathrm{Cr}$ films for high density perpendicular magnetic recording media by using the ECR sputtering, focusing on the effects of Ar gas pressure, and substrate location on the crystallographic and magnetic properties.

\section{EXPERIMENT}

The ECR plasma sputtering deposition apparatus used in this experiment is shown in Fig. 1. The discharge chamber was designed as a $\mathrm{TE}_{113}$ resonance cavity. The main coils are arranged around the periphery of the discharge chamber. The magnetic field of $875 \mathrm{G}$ satisfying the ECR condition is generated in this chamber by these coils. The $2.45 \mathrm{GHz}$ microwave was introduced into the chamber perpendicularly to the chamber sidewall through the waveguide. This waveguide/ discharge chamber geometry enables the continuous deposition of the conductive films. Under the ECR condition, high density plasma is generated at $\mathrm{Ar}$ gas pressure as low as $10^{-2}$ $\mathrm{Pa}$. In this discharge chamber/substrate configuration shown in this figure, the plasma stream occurs from the discharge chamber toward the substrate along the magnetic lines of force. A cylindrical target to which the negative voltage, $V_{a}$, of $-100 \mathrm{~V}$ was applied, was placed at the end of the discharge chamber. The composition of the target was $\mathrm{Co}_{80} \mathrm{Cr}_{20}$ wt \%. The input microwave power was $300 \mathrm{~W}$. The Co-Cr films with a thickness from 0.025 to $0.35 \mu \mathrm{m}$ were deposited on the $30 \mu \mathrm{m}$ thick polyimide substrate at the substrate temperature $T_{\text {sub }}$, of $100^{\circ}$ in centigrade. The Ar gas pressure was varied in the range from $3 \times 10^{-2}$ to $9 \times 10^{-2}$

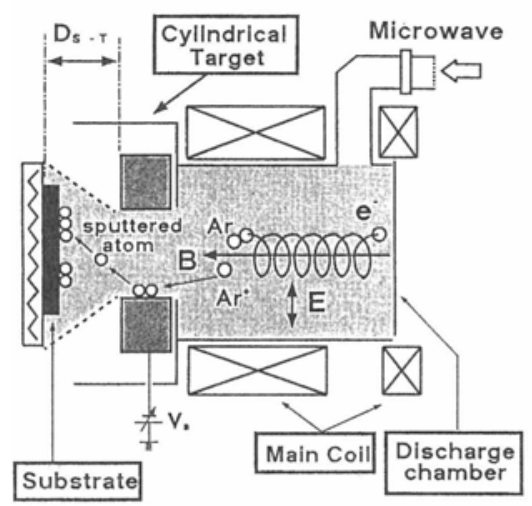

FIG. 1. Cross-sectional view of ECR plasma sputtering deposition apparatus. 


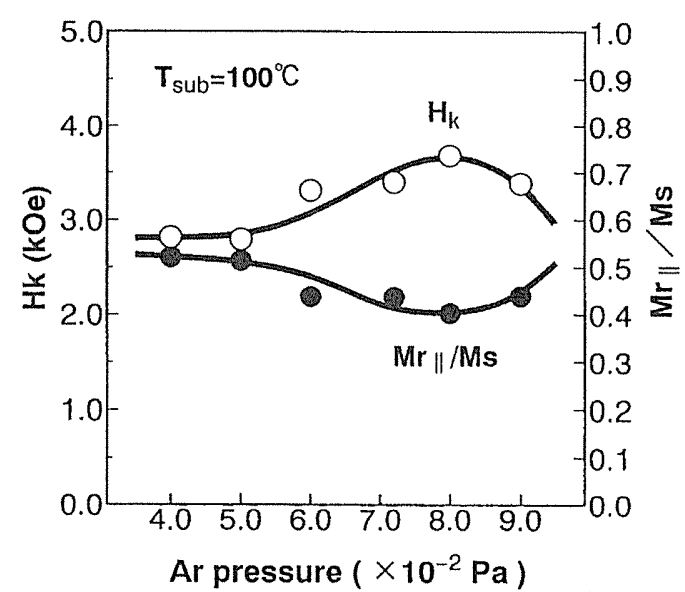

FIG. 2. Ar gas pressure dependence of perpendicular magnetic anisotopy field $H_{k}$, and in-plane squareness $M_{r_{\|}} / M_{s}$.

Pa. The substrate-to-target distance $D_{s-t}$ was varied from 170 to $230 \mathrm{~mm}$. The plasma with the electron temperature from 5 to $12 \mathrm{eV}$, and the electron density from $0.6 \times 10^{9}$ to $1.6 \times 10^{9} \mathrm{~cm}^{-3}$, was achieved in the above described experimental condition. The deposition rate in our experiments varies from 1.5 to $7.2 \mathrm{~nm} / \mathrm{min}$ according to Ar gas pressure and target to substrate distance.

The crystallographic and magnetic properties were measured using an x-ray diffractometer and a vibrating sample magnetometer, respectively.

\section{RESULTS AND DISCUSSION}

At first, the Ar sputtering gas pressure dependence of crystallographic and magnetic properties was investigated for a substrate-to-target distance $D_{s-t}$ of $170 \mathrm{~mm}$. As the $\mathrm{Ar}$ gas pressure increased from $4 \times 10^{-2}$ to $8 \times 10^{-2} \mathrm{~Pa}$, the
$\mathrm{Co}(002) \mathrm{x}$-ray peak intensity drastically increased, and the half-value width of the rocking curve $\Delta \theta_{50}$, became a half, showing that the crystallinity and perpendicular orientation of Co $c$ axis has been improved. The Ar gas pressure dependence of perpendicular magnetic anisotropy field $H_{k}$, and in-plane squareness, $M_{r_{\|}} / M_{s}$, are shown in Fig. 2. The maximum $H_{k}$ and the minimum $M_{r_{\|}} / M_{s}$ were obtained at an Ar gas pressure of $8 \times 10^{-2} \mathrm{~Pa}$. The maximum of perpendicular coercivity $H_{c_{\perp}}$, and the minimum of in-plane coercivity $H_{c_{\|}}$, were also achieved at Ar gas pressure of $8 \times 10^{-2} \mathrm{~Pa}$. These results show that the highly perpendicularly oriented $\mathrm{Co}-\mathrm{Cr}$ films with high perpendicular orientation and magnetic properties desired for high density perpendicular recording have been prepared at relatively high Ar gas pressure of $8 \times 10^{-2} \mathrm{~Pa}$ using ECR sputtering.

To clarify this phenomenon, the probe diagnosis for the ECR plasma was performed. From the $V-I$ characteristics, it was found that both the floating potential of the substrate $V_{f}$, and the plasma potential $V_{s}$, decrease with increasing Ar gas pressure. Some of the Ar ions in the plasma are accelerated by the voltage difference between $V_{s}$ and $V_{f}$, i.e., $V_{s}-V_{f}$, and move along the magnetic lines of force, and finally bombard the substrate. It is surmised that the excessive amount of Ar ion bombardment to the substrate at low Ar gas pressure disturbs the crystal orientation, and consequently, the $H_{k}$ and $H_{c_{\perp}}$ are low.

In the next step, optimization of the substrate-to-target distance $D_{s-t}$ was performed at an Ar gas pressure of $8 \times 10^{-2} \mathrm{~Pa}$. The $D_{s-t}$ was varied from 170 to $230 \mathrm{~mm}$. The $\mathrm{Co}-\mathrm{Cr}$ films with half-value width of the rocking curve $\Delta \theta_{50}$, less than $4^{\circ}$ were obtained around $D_{s-t}$ of 170 and 230 $\mathrm{mm}$. However, the in-plane $\mathrm{M}-\mathrm{H}$ hysteresis loops of the films are different in each other as shown in Fig. 3. The in-plane $\mathrm{M}-\mathrm{H}$ loop for the film deposited at $D_{s-t}$ of $170 \mathrm{~mm}$
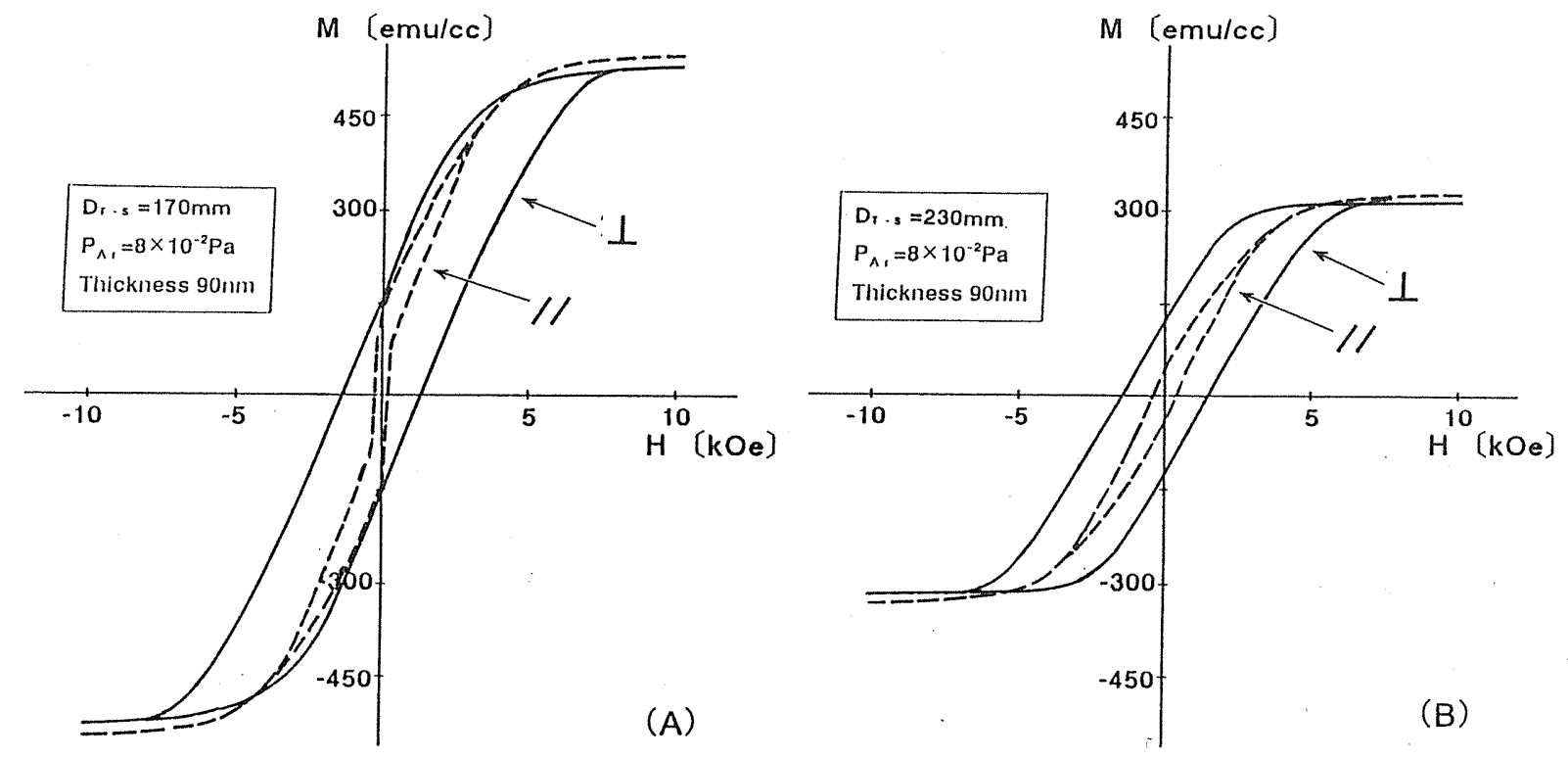

FIG. 3. $\mathrm{M}-\mathrm{H}$ hysteresis loops of $\mathrm{Co}-\mathrm{Cr}$ films in perpendicular direction (solid line) and in-plane direction (dashed line); (a) is for deposition at substrateto-target distance $D_{s-t}$, of $170 \mathrm{~mm}$, and (b) for deposition at $D_{s-t}$ of $230 \mathrm{~mm}$. 


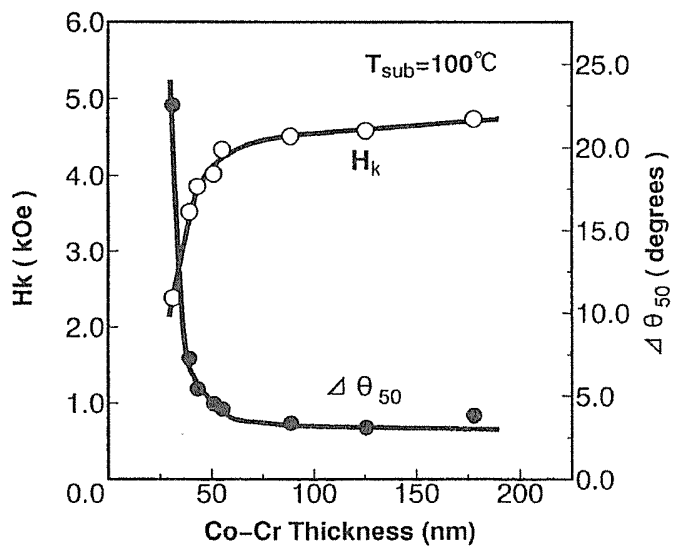

FIG. 4. Thickness dependence of $\Delta \theta_{50}$ and $H_{k}$ of $\mathrm{Co}-\mathrm{Cr}$ films deposited at optimal condition, i.e., $D_{s-t}$ of $230 \mathrm{~mm}$ and $\mathrm{Ar}$ gas pressure of $8 \times 10^{-2} \mathrm{~Pa}$.

has jump at low magnetic field as shown by the broken line. This jump is caused by the existence of a low coercivity initial layer in which the Co $c$ axis does not align in perpendicular direction. ${ }^{11,12}$ In the conventional diode sputtering methods, it is difficult to eliminate this nonoriented initial layer unless the underlayer such as $\mathrm{Ti}$ and $\mathrm{Cr}$ are introduced. On the other hand, for the films deposited by the ECR sputtering at $D_{s-t}$ of $230 \mathrm{~mm}$, no jumps are observed as shown by the solid line because the Ar ion bombardment during deposition is so small that the crystallinity and crystal orientation are not disturbed.

The thickness dependence of the $\Delta \theta_{50}$ and $H_{k}$ is shown in Fig. 4. These Co-Cr films are deposited at $D_{s-t}$ of 230 $\mathrm{mm}$ and at an $\mathrm{Ar}$ gas pressure of $8 \times 10^{-2} \mathrm{~Pa}$. As the $\mathrm{Co}-\mathrm{Cr}$ thickness increases from 27 to $50 \mathrm{~nm}$, the $\Delta \theta_{50}$ drastically decreased and reached a near plateau value of around $3^{\circ}$ at a thickness of $90 \mathrm{~nm}$. $H_{k}$ increased sharply in the thickness range 27 to $50 \mathrm{~nm}$ and showed a relatively small increase about $90 \mathrm{~nm}$. Surprisingly, very small $\Delta \theta_{50}$ of $3.1^{\circ}$ was obtained at a thickness of $90 \mathrm{~nm}$.

The thickness dependence of the perpendicular coercivity, $H_{c_{\perp}}$, is shown in Fig. 5. The open circles are for the films

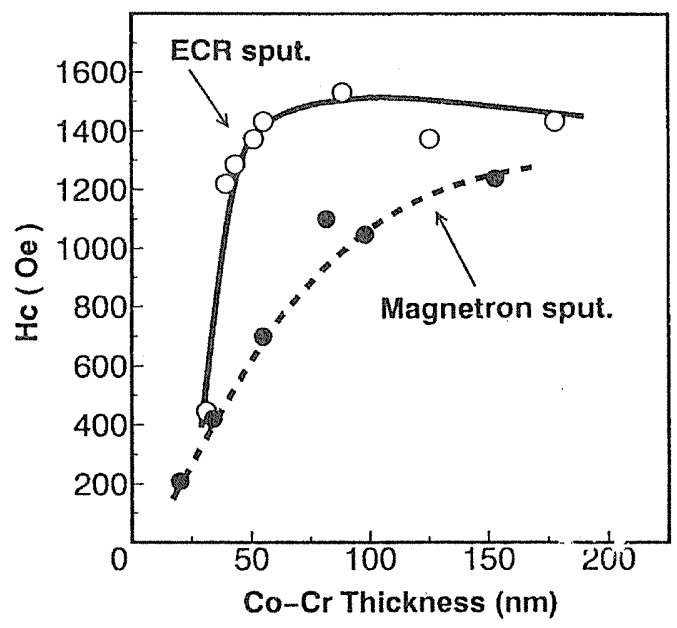

FIG. 5. Perpendicular coercivity $H_{c_{1}}$, as a function of $\mathrm{Co}-\mathrm{Cr}$ film thickness. Open circles are for ECR sputtering deposition, and solid circles are for magnetron diode sputtering deposition. deposited by the ECR sputtering condition tuned at $D_{s-t}$ of $230 \mathrm{~mm}$ and at Ar gas pressure of $8 \times 10^{-2} \mathrm{~Pa}$. For comparison, the data for the $\mathrm{Co}-\mathrm{Cr}$ films prepared by magnetron diode sputtering at the optimal sputtering condition, i.e., Ar gas pressure of $5 \times 10^{-1} \mathrm{~Pa}$, are plotted by solid circles. In both sputtering depositions, the same polyimide substrate was used, and the underlayers which play a role to control the Co crystal orientation were not introduced. Although the films prepared by conventional magnetron sputtering needs at least $100 \mathrm{~nm}$ thickness to achieve perpendicular coercivity higher than $1000 \mathrm{Oe}$, in the ECR sputtering, only $50 \mathrm{~nm}$ thickness is enough to realize 1400 Oe coercivity. This is considered by the authors as the specific advantage of the ECR sputtering over the diode sputtering for the $\mathrm{Co}-\mathrm{Cr}$ films for perpendicular recording.

\section{CONCLUSION}

The ECR sputtering deposition technique was used in the fabrication of the $\mathrm{Co}-\mathrm{Cr}$ perpendicular magnetic recording media. The Co-Cr films with superior crystallinity, perpendicular orientation, and mag tic properties desirable for high density recording were acheved at a high Ar gas pressure of $8 \times 10^{-2} \mathrm{~Pa}$. The Co-Cr films deposited at a target to a substrate distance of $230 \mathrm{~mm}$ showed good crystal orientation $\left(\Delta \theta_{50}\right.$ less than $\left.4^{\circ}\right)$ high perpendicular magnetic anisotropy $\left(H_{k}\right.$ higher than $\left.4 \mathrm{kOe}\right)$, and high perpendicular coercivity over 1400 Oe even when the Co-Cr thickness was as small as about $50 \mathrm{~nm}$. We conclude that the ECR sputtering has high potential in the deposition of the $\mathrm{Co}-\mathrm{Cr}$ films for ultrahigh density recording media.

\section{ACKNOWLEDGMENTS}

We are grateful to Dr. S. Hirono of NTT Interdisciplinary Research Laboratories for his valuable discussion and advice, and T. Nakamura for his experimental assistance in VSM measurements. This research was supported by a Grant-in-Aid for Scientific Research (C) and by the Electric Technology Research Foundation of Chugoku.

${ }^{1}$ S. Iwasaki and Y. Nakamura, IEEE Trans. Magn. MAG-13, 1272 (1977).

${ }^{2}$ S. Yamamoto, Y. Nakamura, and S. Iwasaki, IEEE Trans. Magn. MAG-23, 2070 (1987).

${ }^{3}$ S. Yanase, T. Kiya, N. Honda, and K. Ouchi, Tech. Rep. IEICE MR95-14, 15 (1995).

${ }^{4}$ Y. Maeda, S. Hirono, and M. Asahi, Jpn. J. Appl. Phys. 24, L951 (1985).

${ }^{5}$ Y. Maeda and M. Asahi, J. Appl. Phys. 61, 1972 (1987).

${ }^{6}$ Y. Maeda, K. Takei, S. Yamamoto, and Y. Nakamura, J. Magn. Soc. Jpn. 15, 457 (1991).

${ }^{7}$ T. Ono et al., Jpn. J. Appl. Phys. 23, L534 (1984).

${ }^{8}$ M. Matsuoka and K. Ono, J. Vac. Sci. Technol. A 6, 25 (1988).

${ }^{9}$ C. Takahashi, M. Kiuchi, K. Ono, and S. Matsuo, J. Vac. Sci. Technol. A 6, 2348 (1988).

${ }^{10} \mathrm{~S}$. Hirono, M. Igarashi, and Y. Maeda, Tech. Rep. IEICE MR94-60, 41 (1994).

${ }^{11}$ Y. Nakamura and S. Iwasaki, IEEE Trans. Magn. MAG-23, 153 (1987).

${ }^{12}$ M. Yasumura, K. Ouchi, and S. Iwasaki, IEEE Trans. Magn. MAG-23, 2794 (1987). 